\title{
Aberrant GRK6 promoter methylation is associated with poor prognosis in hypopharyngeal squamous cell carcinoma
}

\author{
XIAOXIA QIU ${ }^{1}$, JIANQIU CHEN ${ }^{3}$, ZHENXIN ZHANG $^{1}$, YIWEN YOU ${ }^{1}$ and ZHIWEI WANG ${ }^{2}$ \\ Departments of ${ }^{1}$ Otorhinolaryngology/Head and Neck Surgery, ${ }^{2}$ General Surgery, Affiliated Hospital \\ of Nantong University, Nantong, Jiangsu 226001; ${ }^{3}$ Department of Otolaryngology Head and Neck Surgery, \\ General Hospital of Jinan Military Region, Jinan, Shandong 250031, P.R. China
}

Received October 6, 2015; Accepted November 8, 2015

DOI: $10.3892 / o r .2015 .4469$

\begin{abstract}
Hypopharyngeal squamous cell carcinoma (HSCC) is one of the most common head and neck cancers with high invasiveness and poor prognosis. To identify targeted therapeutics against metastasis, a better understanding of the regulation of HSCC cell invasion is needed. In recent years, G protein-coupled receptor kinases (GRKs) have been implicated in cancer metastasis through phosphorylation of the activated form of $\mathrm{G}$ protein coupled receptors (GPCRs). However, there is little information regarding GRKs expression in HSCC. In the present study, we examined GRK6 expression in HSCC and also assessed the possible cause of its aberrant expression, as well as its clinical significance. Real-time quantitative PCR (qPCR) and western blotting were performed to analyze the expression of GRK6 in HSCC tissues and corresponding non-malignant tissues. Subsequently, paired HSCC and corresponding non-malignant tissues were evaluated for the methylation status of GRK6 gene promoter using methylationspecific PCR (MSP). Furthermore, we investigated the methylation status and the clinicopathological significance of GRK6 in 45 paired HSCC and corresponding non-malignant tissues. The suppression of GRK6 in hypopharyngeal cell line FaDu by GRK6-shRNA lentivirus transfection was utilized to detect the role of GRK6 in hypopharyngeal cancer progression. Our results showed that the expression of GRK6 mRNA and protein was significantly lower in HSCC than in corresponding adjacent non-tumor tissues, and this downregulation
\end{abstract}

Correspondence to: Professor Zhiwei Wang, Department of General Surgery, Affiliated Hospital of Nantong University, No. 20 Xisi Road, Nantong, Jiangsu 226001, P.R. China

E-mail: youyi701@163.com

Abbreviations: GRKs, G protein-coupled receptor kinases; HSCC, hypopharyngeal squamous cell carcinoma; MSP, methylationspecific PCR; qPCR, real-time quantitative PCR

Key words: GRK6, DNA methylation, hypopharyngeal squamous cell carcinoma, prognosis was found to be in accordance with aberrant methylation of the gene. Hypermethylation of the gene was observed in $77.8 \%$ (35/45) of the HSCC tissues, while it was found in only $42.2 \%$ (19/45) of the corresponding non-malignant tissues. GRK6 methylation was related to depth of tumor invasion and TNM stage. Upon treatment with 5-aza-2'-deoxycytidine, GRK6 expression was upregulated in FaDu cells, and cell invasion was signinficantly inhibited. Furthermore, the suppression of GRK6 by shRNA transfection enhanced FaDu cells invasion. Our results indicate that the aberrant methylation of GRK6 gene promoter may underlie its downregulation in HSCC, and may play an important role in the metastasis of HSCC.

\section{Introduction}

Hypopharyngeal squamous cell carcinoma (HSCC), a malignant neoplasm arising from the outer layer (epithelium) of the hypopharynx, is a subset of head and neck cancer with particularly poor prognosis and more aggressive behavior (1). Over the last decades, although diagnosis and treatment methods including surgical resection, radiation therapy, and neoadjuvant chemotherapy for HSCC are continuously improving, the 5-year survival rate of patients with HSCC is only $\sim 25-40 \%$ (2). Understanding the molecular pathophysiology of HSCC is essential for determining how to effectively inhibit tumor progression and metastasis. Therefore, to identify targeted therapeutics against progression and metastasis, a better understanding cellular and molecular mechanism of the regulation of HSCC cell invasion is needed.

$\mathrm{G}$ protein-coupled receptor kinases (GRKs) are a family of serine/threonine protein kinase that phosphorylate Ser/Thr residues in the cytoplasmic domains and tails of activated G protein-coupled receptors (GPCRs). Receptor phosphorylation triggers a cascade of events that includes the recruitment of arrestins to bind to the phosphorylated Ser/Thr residues of GPCRs, receptor internalization and precluding $\mathrm{G}$ protein coupling, thereby preventing reactivation of the signaling pathway $(3,4)$. Although GRKs have a crucial function in desensitization, maladaptive GRK activity has been implicated in a range of human diseases, including heart failure, opiate addiction and tumor progression and metastasis $(3,5,6)$. Therefore, GRKs are considered important therapeutic targets (4-6). GRK6 is expressed in many human 
tissues, and participates in the pathological process of many diseases (7-10). Recently, some studies reported GRK6 also has been implicated in various cancer metastasis, including lung cancer (11), medulloblastoma (12) and multiple myeloma (10). However, the specific roles and regulation of GRK6 in HSCC have not been investigated.

In the present study, we investigated GRK6 expression in HSCC and also assessed the possible cause of its aberrant expression. In addition, we assessed the GRK6 gene promoter methylation status in HSCC cell lines and HSCC tissues, and evaluated its clinical significances. The role of GRK6 might provide new insights into the tumorigenesis of HSCC.

\section{Materials and methods}

Human tissue specimens. A total of 45 pairs of primary hypopharyngeal squamous cell carcinoma (HSCC) and corresponding non-malignant tissue specimens were obtained from patients with HSCC at the Affiliated Hospital of Nantong University and General Hospital of Jinan Military Region between July 2003 and June 2011. Clinical characteristics of HSCC patients were extracted from their medical records, including: gender, age, histological type, differentiation grade and tumor stage. None of the cancer patients received any type of treatment (radiation therapy, chemotherapy or immunotherapy) before surgery. Specimens were collected immediately after tumor excision during surgery. All specimens were subjected to histological diagnosis by a pathologist. The study protocol was approved by the Human Research Ethics Committee of the Affiliated Hospital of Nantong University and General Hospital of Jinan Military Region, and all patients provided written informed consent prior to participation in the study.

Cell culture. The human HSCC cell line, FaDu cells, was purchased from the American Type Culture Collection (ATCC; Manassas, VA, USA). FaDu cells were cultured in Dulbecco's modified Eagle's medium (DMEM; Invitrogen, Carlsbad, CA, USA) supplemented with $10 \%$ fetal bovine serum (FBS; Invitrogen) at $37^{\circ} \mathrm{C}$ in a humidified incubator with $5 \% \mathrm{CO}_{2}$.

RNA extraction and real-time quantitative PCR ( $q P C R)$. Total RNA was extracted from FaDu cells, human HSCC tissues, and corresponding non-malignant tissues from the same patients. Extraction was done using with TRIzol reagent, as described by the manufacturer (Invitrogen). One microgram of DNase I-treated (Fermentas, Vilnius, Lithuania) total RNA were converted to cDNA using a Reverse Transcription System kit (Fermentas, Burlington, ON, Canada). The GRK6 mRNA expression level was determined by following Maxima SYBR Green/ROX qPCR Master Mix kit (Fermentas) with the following primer specific either for GRK6 or the housekeeping gene GAPDH (internal control). GRK6: 5'-AAAAC ACCTTCAGGCAATACCG-3' (sense) and 5'-AGGCCAAGC TCACTACAAACCTA-3' (antisense); GADPH: 5'-CATGAG AAGTATGACAACAGCCT-3' (sense) and 5'-AGTCCTT CCACGATACCAAAGT-3' (antisense). As a negative control we used DEPC-treated water to replace cDNA templates for every PCR. The relative quantitative value was expressed by the $2^{-\Delta \Delta \mathrm{Ct}}$ method.
Protein isolation and western blotting. Human HSCC tissues and their corresponding non-malignant tissues were homogenized in RIPA lysis buffer, and the lysates were cleared by centrifugation $(12,000 \mathrm{rpm})$ at $4^{\circ} \mathrm{C}$ for $20 \mathrm{~min}$, and denatured by boiling for $5 \mathrm{~min}$. Equal amounts of protein $(30 \mu \mathrm{g})$ were electrophoresed in $10 \%$ polyacrylamide gels. Subsequently, the proteins were transferred to PVDF membranes (Millipore, Bedford, MA, USA). The membranes were blocked with $5 \%$ non-fat dry milk in TBST buffer for $1 \mathrm{~h}$ at room temperature and incubated with a rabbit polyclonal anti-human GRK6 antibody (1:300; Santa Cruz Biotechnology, Inc., Santa Cruz, CA, USA) or an anti-GAPDH (internal control) at $4^{\circ} \mathrm{C}$ overnight at room temperature. The next day, the membranes were washed three times with TBST, after which they were incubated for $2 \mathrm{~h}$ with a 1:2,000 dilution of secondary antibodies. After washing, immunoreactive protein bands were visualized using an electrochemiluminescence (ECL) detection kit (Thermo Fisher Scientific Inc., Rockford, IL, USA). Protein bands were scanned and quantified using a Gel Image Analysis System. Each experiment was repeated three times. The protein levels were normalized to that of GAPDH.

Genomic DNA isolation and MSP. Genomic DNA was extracted from FaDu cell line and primary HSCC tissues through SDS/proteinase $\mathrm{K}$ treatment, followed by phenolchloroform extraction and ethanol precipitation. Bisulfite treatment was performed using the EZ DNA Methylation-Gold kit (Zymo Research, Irvine, CA, USA), according to the manufacturer's instructions. http://www.urogene.org/cgi-bin/ methprimer/methprimer.cgi was used to analyze the $3000 \mathrm{bp}$ region of the GRK6 gene promoter region (-2000 bp to $+1000 \mathrm{bp}$ ) for the presence of $\mathrm{CpG}$ islands. After prediction analysis, we found two $\mathrm{CpG}$ islands ( -855 to -318 and -310 to +475). The GRK6 methylation-specific primers used were 5'-TGGGTGGAGAGAAATTATAATATTC-3' (sense) and 5'-TATAACCGTATTCCCTTAAATCGAC-3' (antisense), $167 \mathrm{bp}$; and the unmethylation-specific primers used were 5'-GGTGGAGAGAAATTATAATATTTGG-3' (sense) and 5'-TATAACCATATTCCCTTAAATCAAC-3' (antisense), $165 \mathrm{bp}$. The reaction mixture contained $1 \mu \mathrm{l}$ DNA in a volume of $50 \mu \mathrm{l}$ containing $1 \mu \mathrm{l}$ of each primer, $2 \mathrm{X}$ GC buffer I, $2.5 \mathrm{mM}$ dNTP Mix and 2.5 U LA Taq (Takara). The MSP conditions were as follows: $94^{\circ} \mathrm{C}$ for $5 \mathrm{~min}, 40$ cycles of $94^{\circ} \mathrm{C}$ for $30 \mathrm{sec}, 54^{\circ} \mathrm{C}$ for $30 \mathrm{sec}, 72^{\circ} \mathrm{C}$ for $45 \mathrm{sec}$ and $72^{\circ} \mathrm{C}$ for $10 \mathrm{~min}$. Water was run as a negative control in every MSP reaction. All procedures were repeated three times. The PCR products were subjected to $2 \%$ agarose gel electrophoresis at $100 \mathrm{~V}$ for $30 \mathrm{~min}$.

Treatment of cells with 5-Aza-dC. HSCC cells (FaDu) were seeded at a density of $5 \times 10^{5}$ cells/well in 6 -well culture plates in DMEM (Invitrogen) containing $10 \% \mathrm{FBS}$, and incubated in a humidified atmosphere of $5 \% \mathrm{CO}_{2}$ at $37^{\circ} \mathrm{C}$. After overnight culture, cells were incubated with medium containing 0,5 and $10 \mu \mathrm{mol} / 1$ 5-Aza-2'-deoxycytidine (5-Aza-dC) (Sigma, St. Louis, MO, USA) for 3 days. Total RNA was isolated after treatment, and mRNA was analyzed by qPCR as described above. 

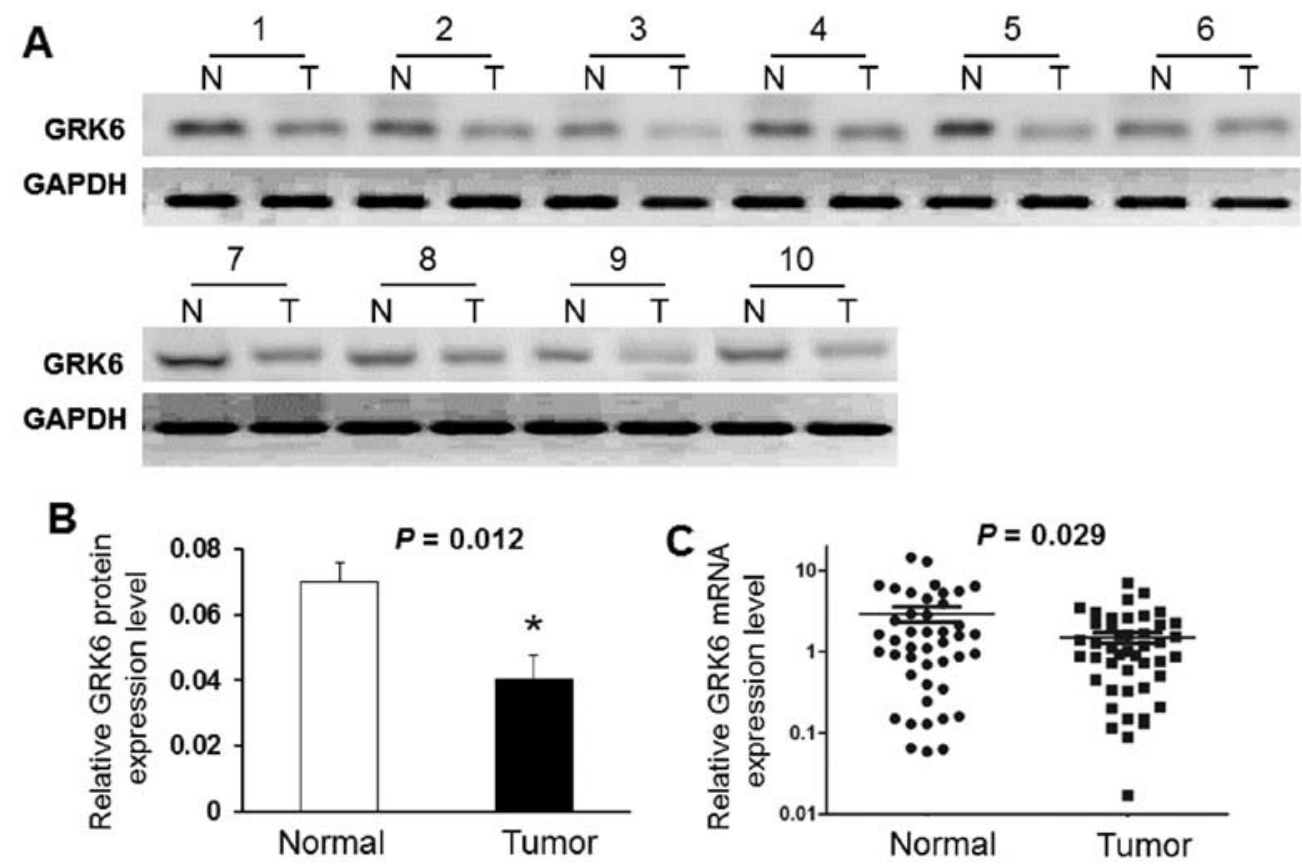

Figure 1. GRK6 gene expression was downregulated in HSCC tissues. (A) GRK6 protein expression was downregulated in HSCC tissues. Representative western blot analysis of GRK6 expression in HSCC and corresponding non-tumor tissue specimens are showed. (B) Summary of the western blot results from 35 HSCC tissues ( $\mathrm{T}$ ) and corresponding non-tumor tissues $(\mathrm{N})$ presented as relative bands density normalized to the $\beta$-actin of the same samples. (C) GRK6 mRNA expression level in HSCC tissues and matched adjacent non-tumor tissues were determined by real-time RT-PCR and was normalized to GAPDH.

Lentivirus-mediated shRNA knockdown of GRK6 expression. The target sequence of GRK6 siRNA sequence (5'-GCCGA CUACCUCGACAGCAUCUACU-3') was used as previously described (13), a scrambled sequence (5'-TTCTCCGAACG TGTCACGT-3') was used as negative control for RNA interference (RNAi), which had no significant homology to any human gene sequences. Inverted and self-complementary hairpin DNA oligos targeting GRK6 mRNA were obtained from Genchem Biotechnology Co. (Shanghai, China). The stem-loop oligonucleotides were synthesized and cloned into a lentivirus-based vector pGCSIL-GFP and resulting plasmids were named as GRK6-shRNA and scrambled shRNA respectively. Lentiviral vector production was performed according to standard Invitrogen protocol. Then the lentiviral titers of GRK6-shRNA and scrambled shRNA were identified. Experiments for lentivector transduction were carried out as follows: human HSCC cell line $\mathrm{FaDu}$ were plated at $5 \times 10^{5}$ cells in 6-well culture plates, and transducted with scrambled shRNA, GRK6-shRNA lentivirus at a multiplicity of infection (MOI) of 20 in the presence of $8 \mathrm{mg} / \mathrm{ml}$ of polybrene. After $24 \mathrm{~h}$, the medium was replaced with fresh DMEM containing $10 \%$ FBS and the cells were cultured for another $48 \mathrm{~h}$. Cells were harvested at $72 \mathrm{~h}$ after infection and the knockdown efficiency of GRK6 was evaluated by quantitative real-time PCR and western blot analysis.

Transwell chamber invasion assay. The human HSCC cell line, FaDu cells, incubated with 5-Aza-dC for 3 days or transfected with GRK6-shRNA lentivirus were separately detached from the culture plates, then seeded onto Matrigel-coated $8 \mu \mathrm{m}$ pore size Transwell filters (Corning Life Sciences, Corning, $\mathrm{NY}, \mathrm{USA}$ ) in the upper well at the density of $5 \times 10^{4}$ per in $200 \mu \mathrm{l}$ of culture medium (DMEM containing $1 \%$ fetal calf serum), in the lower chamber, $500 \mu$ l DMEM containing $10 \%$ FBS was added as a chemoattractant. After $24 \mathrm{~h}$ of incubation, non-invading cells on the upper wells were removed with a cotton-tipped swab. Then, the lower side of the filters were fixed in $4 \%$ paraformaldehyde for $10 \mathrm{~min}$, and stained with $0.1 \%$ crystal violet for $30 \mathrm{~min}$. The invaded cells were viewed under a microscope and counted in five fields of view at x200 magnification. The invasion ability of the cancer cells was expressed as the mean number of cells in five fields. The assay was performed in three independent experiments.

Statistical analysis. Statistical analyses were performed using SPSS 18.0 software (SPSS, Inc., Chicago, IL, USA). We used the $\chi^{2}$ test for clinicopathological features, and the Student's t-test or one-way ANOVA test for continuous variables. Data are expressed as mean $\pm \mathrm{SD}$. $\mathrm{P}<0.05$ was considered statistically significant.

\section{Results}

GRK6 expression is downregulated in HSCC tissues. We investigated GRK6 protein and mRNA levels in the hypopharyngeal squamous cell carcinoma (HSCC) specimens and corresponding non-malignant tissues by western blotting and quantitative real-time PCR. Western blot analysis showed that the expression level of GRK6 protein was significantly decreased in 18 of 25 paired HSCC tissues compared with the corresponding adjacent non-malignant tissues, and ten cases of the representative western blotting results are shown in Fig. 1A. Moreover, the average GRK6 protein level in HSCC tissues was significantly lower than that of GRK6 in adjacent non-malignant tissues ( $\mathrm{P}=0.012$; Fig. $1 \mathrm{~B})$. In addition, the results of quantitative RT-PCR revealed that GRK6 mRNA 


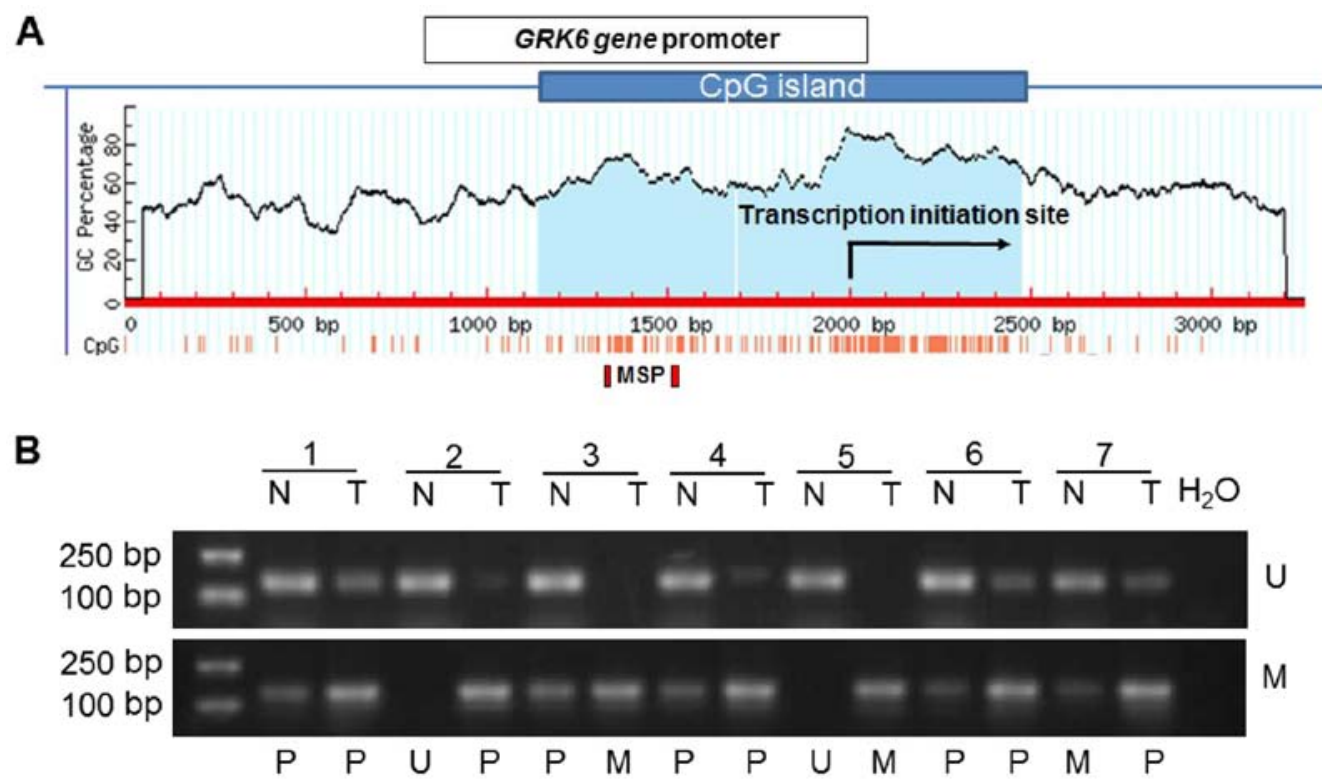

Figure 2. MSP show DNA methylation of GRK6 in HSCC tissues. (A) Schematic of two CpG islands (-855 to -318 and -310 to +475) in GRK6 gene promoter area $(-2000 \mathrm{bp}$ to $+1000 \mathrm{bp})$. (B) Representative examples of the methylation analysis of GRK6 in HSCC tissues and their corresponding non-tumor tissues. T, tumor tissue; $\mathrm{N}$, non-tumor tissue; $\mathrm{U}$, unmethylation; $\mathrm{M}$, methylation; $\mathrm{P}$, partial methylation; $\mathrm{H}_{2} \mathrm{O}$, positive control.

A

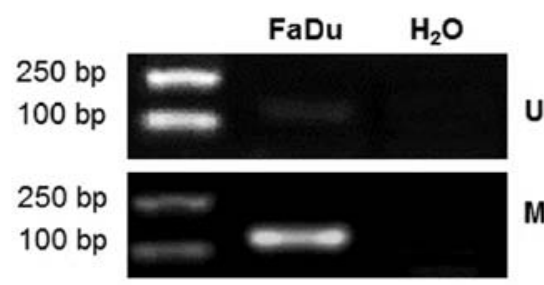

C

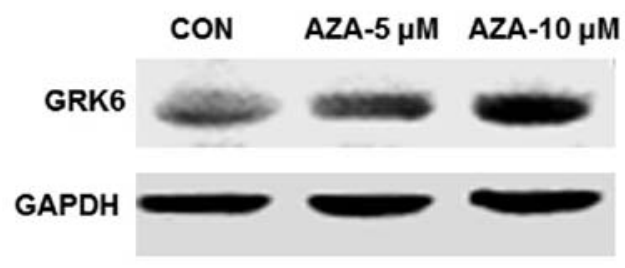

B

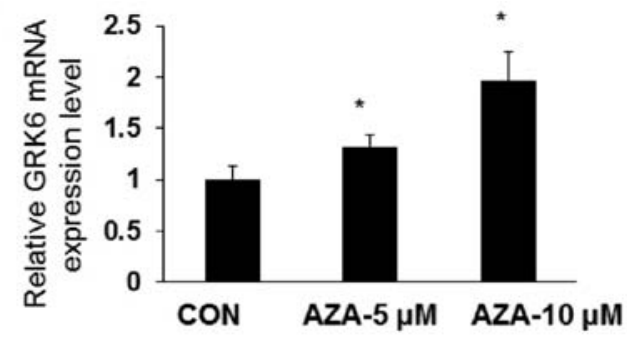

D

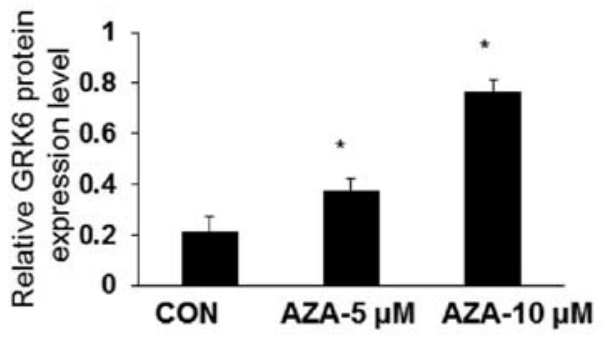

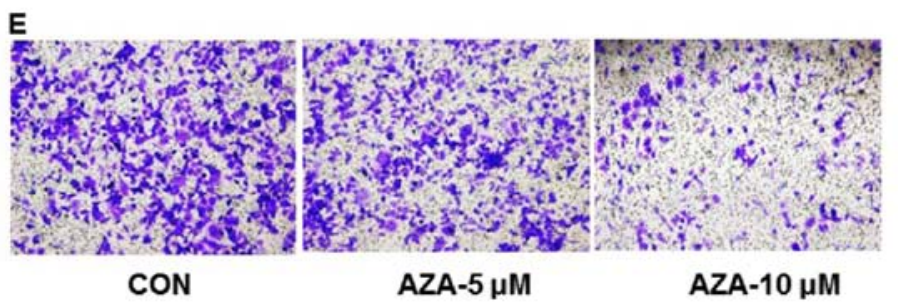

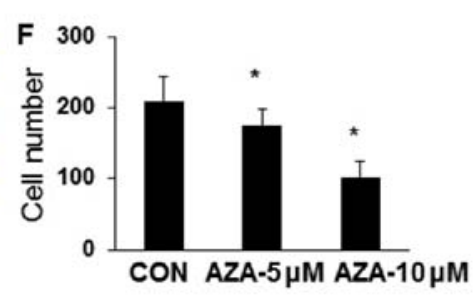

Figure 3. Downregulation of GRK6 in FaDu cells is associated with methylation of GRK6. (A) DNA methylation of GRK6 in $\mathrm{H}_{2} \mathrm{O}$ and FaDu cell lines. GRK6 was hypermethylated in FaDu cells. (B-D). qRT-PCR and western blot analysis of GRK6 expression after treatment with 5-Aza-dC (5 or $10 \mu \mathrm{M})$ in FaDu cells (E and F) The Matrigel invasion assay was performed to assess the demethylation effect of GRK6 on the invasion ability of FaDu cell lines after treatment with 5-Aza-dC for 3 days. Results showed the number of invading cells was significantly reduced after treatment with 5-Aza-dC compared with the control group. ${ }^{*} \mathrm{P}<0.05$, compared with the control cells without 5-Aza-dC treatment.

expression was downregulated in 32 of 45 paired HSCC samples compared with corresponding non-malignant tissues. The mean expression value of mRNA in HSCC tissues was significantly lower than the value in paired adjacent nonmalignant tissues $(\mathrm{P}=0.029$; Fig. 1C).
Low expression of GRK6 is related to DNA methylation. To identify whether low GRK6 expression was due to DNA methylation, MSP was used to examine DNA methylation status of GRK6 gene promoter CpG islands in 45 paired HSCC specimens and corresponding non-malignant tissues. DNA 

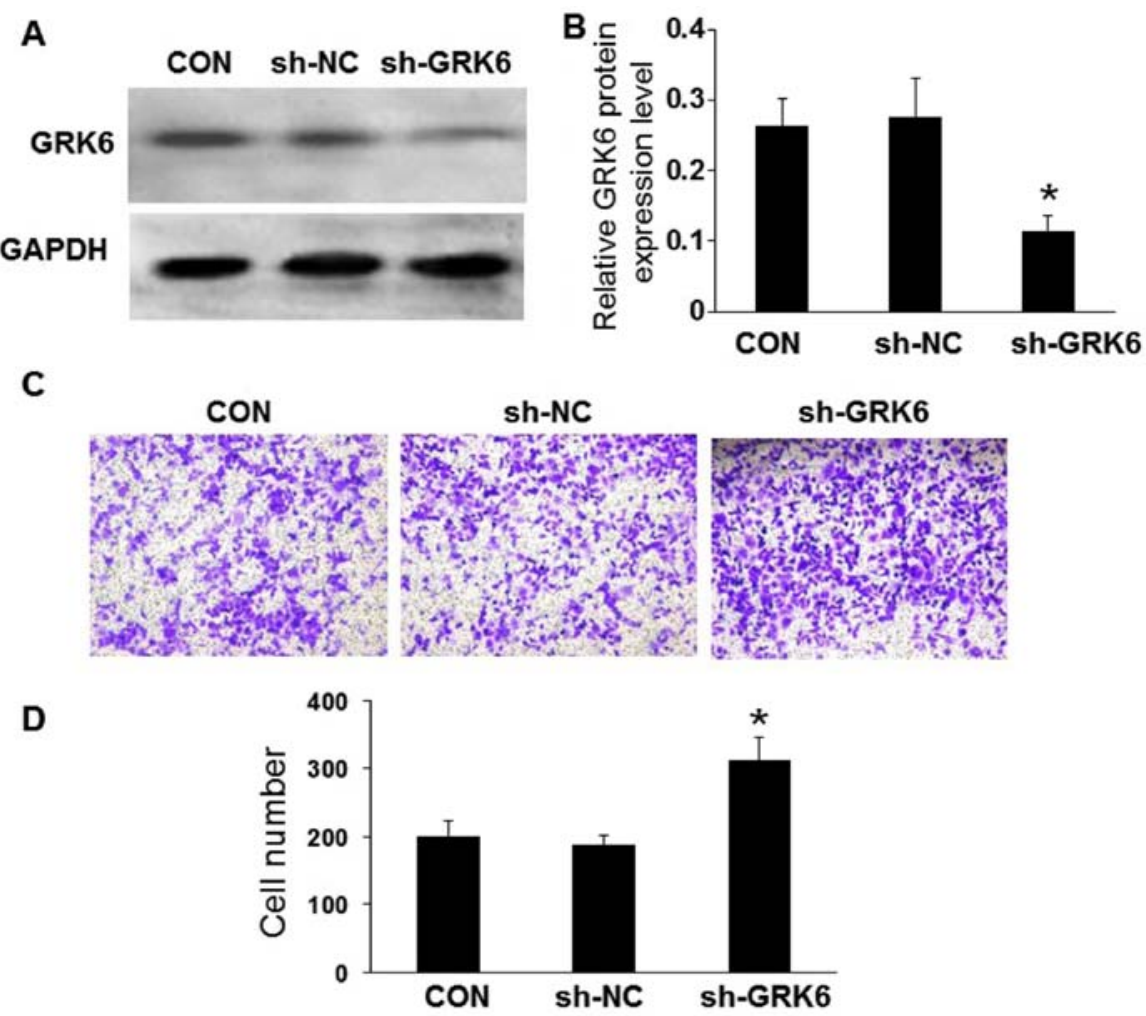

Figure 4. Suppression of GRK6 expression promotes tumor cell invasion. (A and B) GRK6 expression in sh-GRK6 FaDu cells. The level of GRK6 in sh-GRK6, sh-NC and control group was determined by western blotting. (C and D) Invasion assays using sh-NC, sh-GRK6 and control FaDu cells. The invasion ability is presented as fold change in number of cells invaded to the bottom chamber. Bars represent the mean SEM of samples measured in triplicate, and each experiment was repeated at least three times.

Table I. Clinicopathological parameters of HSCC tissue samples and GRK6 methylation.

\begin{tabular}{|c|c|c|c|c|}
\hline \multirow[b]{2}{*}{ Clinical parameters } & \multirow{2}{*}{$\begin{array}{c}\text { Total } \\
(\mathrm{n}=45)\end{array}$} & \multicolumn{2}{|c|}{$\begin{array}{c}\text { GRK6 } \\
\text { methylation }\end{array}$} & \multirow[b]{2}{*}{ P-value } \\
\hline & & M & $\mathrm{U}$ & \\
\hline Gender & & & & 0.869 \\
\hline Male & 17 & 13 & 4 & \\
\hline Female & 28 & 22 & 6 & \\
\hline Age (years) & & & & 0.107 \\
\hline$<60$ & 19 & 17 & 2 & \\
\hline$\geq 60$ & 26 & 18 & 8 & \\
\hline TNM stage & & & & $<0.001^{\mathrm{a}}$ \\
\hline $\mathrm{I} / \mathrm{II}$ & 12 & 5 & 7 & \\
\hline III/IV & 33 & 30 & 3 & \\
\hline Histologic differentiation & & & & 0.370 \\
\hline Well & 8 & 5 & 3 & \\
\hline Moderate & 21 & 16 & 5 & \\
\hline Poor & 16 & 14 & 2 & \\
\hline Lymph node metastasis & & & & $0.003^{\mathrm{a}}$ \\
\hline Positive & 31 & 28 & 3 & \\
\hline Negative & 14 & 7 & 7 & \\
\hline
\end{tabular}

$\mathrm{U}$, unmethylated cases, $\mathrm{M}$, methylated and partial methylated cases. ${ }^{\mathrm{a}} \mathrm{P}<0.05$ was considered statistically significant. methylation occurred in $77.8 \%$ (35/45) of HSCC tissues and $42.2 \%$ (19/45) of non-malignant tissues. No methylation was observed in 22.2\% HSCC tissues and 57.8\% non-malignant tissues. The difference in methylation between the primary HSCC and the non-malignant tissue specimens was significant (Fig. 2; $\mathrm{P}=0.001$ ). Furthermore, DNA methylation of GRK6 was related to TNM stage and lymph node metastasis (Table I). We also examined the DNA methylation status of GRK6 gene promoter in hypopharyngeal cancer cell lines. Data from MSP analysis showed hypermethylation of GRK6 gene promoter in FaDu (Fig. 3A).

Reactivation of GRK6 expression after treatment with 5-Aza-dC. After treatment with the demethylating agent 5-Aza-dC, we first assessed GRK6 mRNA expression in HSCC cell lines and found that the expression of GRK6 mRNA was significantly increased in $\mathrm{FaDu}$, with the highest expression occurring at a concentration of $10 \mu \mathrm{mol} / \mathrm{l}$ (Fig. 3B). We further detected the expression of GRK6 at the protein level after treatment with 5-Aza-dC, and found that GRK6 protein was also significantly elevated, and reached the peak at a concentration of $10 \mu \mathrm{mol} / 1$ (Fig. 3C and D).

Invasion inhibition of FaDu cells after treatment with 5-Aza$d C$. To examine whether the reactivation of GRK6 expression can regulate HSCC invasion, we analyzed the invasion capability of the $\mathrm{FaDu}$ cells using the Matrigel invasion assay as described above. Briefly, after treatment with $10 \mu \mathrm{mol} / 1$ 5-Aza-dC for 3 days, FaDu cells were seeded the upper well 
of Matrigel-coated Transwell filters. After $24 \mathrm{~h}$ of incubation, cells in the lower side of the filters were fixed, stained and counted. The Matrigel invasion assay showed that the number of invading FaDu cells was significantly reduced after 5-Aza-dC treatment compared to the control group, and this reduction was significant $(\mathrm{P}<0.05$; Fig. $3 \mathrm{E}$ and $\mathrm{F})$.

Invasion enhancement of FaDu cells after knocking down GRK6 expression. We used the GRK6-shRNA lentivirus to knock down GRK6 expression in the FaDu cell lines. Realtime quantitative PCR and western blot results confirmed GRK6 was significantly downregulated after 72-h transfection (Fig. 4A and B). Matrigel invasion assay showed the number of invading $\mathrm{FaDu}$ cells was significantly increased after transfection with GRK6-shRNA $(312 \pm 34)$ compared with the scramble shRNA transfected group $(189 \pm 14)$ and the control

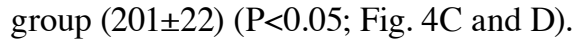

\section{Discussion}

GRK6, the most recently identified member of this family, is a protein of 576 amino acids with a predicted molecular mass of $66 \mathrm{kDa}$ (14). Previously, we observed that decreased GRK6 expression may be involved in the process of pain signals from the affected body as restricted to the areas receiving sensory input from the injured sciatic nerve (15). Previous reported data suggested that GRK6 plays a significant role in the desensitization of the endogenously expressed M3 muscarinic acetylcholine ( $\mathrm{mACh}$ ) receptor in human SH-SY5Y neuroblastoma cells (16). Besides, GRK6 silencing in myeloma cells induced a tumor suppressor effect by inhibiting STAT3 phosphorylation and decreasing tumor cell survival (10). GRK6 is related to the adhesion and motility of several cancer cell lines, and it can significantly inhibit the migration of PC3, MB231 and HeLa cells (17). As integrindependent phosphorylation of kinases, GRK6 was identified for their critical role in cell adhesion and migration possibly through involvement in actin cytoskeleton arrangement (17). Moreover, increased CXCR4 expression is associated with aggressive cancer behavior (18), and GRK6 typically has a negative regulatory role in CXCR4 activation and CXCL12induced cell migration $(19,20)$. Additionally, it is reported that increase in tumor angiogenesis and metastasis observed in GRK6 deficient animals is due to increased production of MMPs mediated by CXCR2, which demonstrated that GRK6 plays a feedback regulatory role in CXCR2-mediated tumor development, invasion and metastasis (21). Furthermore, GRK6 interacts with GIT1 that serves as an integrator and provides a scaffold for the signaling molecules to regulate cell adhesion and cytoskeleton organization (22), and indirectly transactivate EGFR, which in turn affects cancer cell migration and invasion. In addition, GRK6 may affect the migration and invasion of cancer cell through cAMP (23), and calmodulin (24). In the present study, we showed that GRK6 expression was decreased at the mRNA and protein levels, respectively, using qRT-PCR and western blot analysis, in most tumor tissues compared to their adjacent non-malignant tissues, and knock-down of GRK6 expression by LV-shRNA stimulated invasion potential in $\mathrm{FaDu}$ cells by using the matrigel invasion assay.
However, the primary mechanisms of decreased GRK6 expression in tumorigenesis remain largely unclear. We hold the opinion that the methylation of $\mathrm{CpG}$ islands in the promoter region appears to be one major mechanism in HSCC. Hypermethylation has been implicated in the transcriptional repression of many tumor suppressor genes (25). Multiple genes inactivated by promoter $\mathrm{CpG}$ island methylation have been observed in a variety of human cancers, including HSCC (26). Herein, using MSP, we observed that GRK6 was hypermethylated in FaDu cells, and found that the methylation of GRK6 occurred in $77.8 \%$ of HSCC tissues and $42.2 \%$ of non-malignant tissues, while no methylation was observed in $22.2 \%$ HSCC tissues and $57.8 \%$ non-malignant tissues. Thus, inhibiting DNA methylation using demethylating agents led to an increase in expression. As a demethylating agent, 5-aza-2'-deoxycytidine (5-Aza-dC) has been extensively used for epigenetic research. Consequently, silenced genes induced by hypermethylation are re-expressed after treatment with the demethylating agent (27). In the present study, our results showed that GRK6 was significantly upregulated after treatment with 5-Aza-dC, and reactivation of GRK6 expression inhibited the invasion ability of $\mathrm{FaDu}$ cells.

Collectively, GRK6 expression may have significant value as an unfavorable progression indicator for HSCC patients. We provide compelling evidence that attenuated GRK6 expression leads to promotion of cell invasion as a result of DNA methylation, and reactivation of GRK6 expression can suppress cell invasion by using 5 -Aza-dC in FaDu cells.

\section{Acknowledgements}

The present study was supported by grants from the Jiangsu Postdoctoral Science Foundation (1402202C), the Jiangsu 'Six Talent Peaks' Foundation (2014-WSW-030), Nantong Science and Technology Project (HS20140008) and the Project of Affiliated Hospital of Nantong University (TDFY0306).

\section{References}

1. Hall SF, Groome PA, Irish J and O'Sullivan B: The natural history of patients with squamous cell carcinoma of the hypopharynx. Laryngoscope 118: 1362-1371, 2008.

2. Wycliffe ND, Grover RS, Kim PD and Simental A Jr: Hypopharyngeal cancer. Top Magn Reson Imaging 18: 243-258, 2007.

3. Pitcher JA, Freedman NJ and Lefkowitz RJ: G protein-coupled receptor kinases. Annu Rev Biochem 67: 653-692, 1998.

4. Premont RT and Gainetdinov RR: Physiological roles of G protein-coupled receptor kinases and arrestins. Annu Rev Physiol 69: 511-534, 2007.

5. Métayé $\mathrm{T}$, Gibelin $\mathrm{H}$, Perdrisot $\mathrm{R}$ and Kraimps JL: Pathophysiological roles of G-protein-coupled receptor kinases. Cell Signal 17: 917-928, 2005.

6. Dorn GW II: GRK mythology: G-protein receptor kinases in cardiovascular disease. J Mol Med Berl 87: 455-463, 2009.

7. Ahmed MR, Berthet A, Bychkov E, Porras G, Li Q, Bioulac BH, Carl YT, Bloch B, Kook S, Aubert I, et al: Lentiviral overexpression of GRK6 alleviates L-dopa-induced dyskinesia in experimental Parkinson's disease. Sci Transl Med 2: 28ra28, 2010.

8. Eijkelkamp N, Heijnen CJ, Lucas A, Premont RT, Elsenbruch S, Schedlowski $M$ and Kavelaars A: G protein-coupled receptor kinase 6 controls chronicity and severity of dextran sodium sulphate-induced colitis in mice. Gut 56: 847-854, 2007.

9. Murga C and Mayor F Jr: GRK6, a gatekeeper of visceral hyperalgesia. Brain Behav Immun 23: 16-17, 2009.

10. Tiedemann RE, Zhu YX, Schmidt J, Yin H, Shi CX, Que Q, Basu G, Azorsa D, Perkins LM, Braggio E, et al: Kinome-wide RNAi studies in human multiple myeloma identify vulnerable kinase targets, including a lymphoid-restricted kinase, GRK6. Blood 115: 1594-1604, 2010. 
11. Sutton N, Smith N, Thomas AJ, Raghuwanshi SK and Richardson RM: Abstract 2821: GRK6 deficiency promotes tumor aggressiveness and metastasis in a murine model of human lung cancer. Cancer Res 73 (Suppl 8): 2821-2821, 2013.

12. Yuan L, Zhang H, Liu J, Rubin JB, Cho YJ, Shu HK, Schniederjan $\mathrm{M}$ and MacDonald TJ: Growth factor receptor-Srcmediated suppression of GRK6 dysregulates CXCR4 signaling and promotes medulloblastoma migration. Mol Cancer 12: 18, 2013.

13. Hara S, Arawaka S, Sato H, Machiya Y, Cui C, Sasaki A, Koyama $\mathrm{S}$ and Kato T: Serine 129 phosphorylation of membraneassociated alpha-synuclein modulates dopamine transporter function in a $\mathrm{G}$ protein-coupled receptor kinase-dependent manner. Mol Biol Cell 24: 1649-1660, 2013.

14. Benovic JL and Gomez J: Molecular cloning and expression of GRK6. A new member of the $\mathrm{G}$ protein-coupled receptor kinase family. J Biol Chem 268: 19521-19527, 1993.

15. Zhou Y, Huang X, Wu H, Xu Y, Tao T, Xu G, Cheng C and Cao S: Decreased expression and role of GRK6 in spinal cord of rats after chronic constriction injury. Neurochem Res 38: 2168-2179, 2013.

16. Willets JM, Challiss RA and Nahorski SR: Endogenous G protein-coupled receptor kinase 6 regulates M3 muscarinic acetylcholine receptor phosphorylation and desensitization in human SH-SY5Y neuroblastoma cells. J Biol Chem 277: 15523-15529, 2002.

17. Chen Y, Lu B, Yang Q, Fearns C, Yates JR III and Lee JD: Combined integrin phosphoproteomic analyses and small interfering RNA-based functional screening identify key regulators for cancer cell adhesion and migration. Cancer Res 69: 3713-3720, 2009.

18. Teicher BA and Fricker SP: CXCL12 (SDF-1)/CXCR4 pathway in cancer. Clin Cancer Res 16: 2927-2931, 2010.
19. McCormick PJ, Segarra M, Gasperini P, Gulino AV and Tosato G: Impaired recruitment of Grk6 and beta-Arrestin 2 causes delayed internalization and desensitization of a WHIM syndrome-associated CXCR4 mutant receptor. PLoS One 4: e8102, 2009.

20. Vroon A, Heijnen CJ, Raatgever R, Touw IP, Ploemacher RE Premont RT and Kavelaars A: GRK6 deficiency is associated with enhanced CXCR4-mediated neutrophil chemotaxis in vitro and impaired responsiveness to G-CSF in vivo. J Leukoc Biol 75: 698-704, 2004

21. Raghuwanshi SK: GRK6 deficiency promotes angiogenesis, tumor progression and metastasis. J Immunol 190: 896, 2013.

22. Premont RT, Claing A, Vitale N, Freeman JL, Pitcher JA, Patton WA, Moss J, Vaughan M and Lefkowitz RJ: beta2-Adrenergic receptor regulation by GIT1, a G protein-coupled receptor kinase-associated ADP ribosylation factor GTPase-activating protein. Proc Natl Acad Sci USA 95: 14082-14087, 1998.

23. Ribas C, Penela P, Murga C, Salcedo A, García-Hoz C, JuradoPueyo M, Aymerich I and Mayor F Jr: The G protein-coupled receptor kinase (GRK) interactome: Role of GRKs in GPCR regulation and signaling. Biochim Biophys Acta 1768: 913-922, 2007.

24. Pronin AN, Satpaev DK, Slepak VZ and Benovic JL: Regulation of $\mathrm{G}$ protein-coupled receptor kinases by calmodulin and localization of the calmodulin binding domain. J Biol Chem 272: 18273-18280, 1997.

25. Razin A and Riggs AD: DNA methylation and gene function. Science 210: 604-610, 1980.

26. Wei DM, Liu DY, Lei DP, Jin T, Wang J and Pan XL: Aberrant methylation and expression of DAPk1 in human hypopharyngeal squamous cell carcinoma. Acta Otolaryngol 135: 70-78, 2015.

27. Christman JK: 5-Azacytidine and 5-aza-2'-deoxycytidine as inhibitors of DNA methylation: Mechanistic studies and their implications for cancer therapy. Oncogene 21: 5483-5495, 2002. 